\title{
Poids du contexte sur la répartition noms/verbes dans le langage adressé à l'enfant en français et en turc
}

\author{
Sophie Kern*, Florence Chenu*, Feyza Turkay ${ }^{\circ}$ \\ *Laboratoire Dynamique Du Langage, CNRS \& Université de Lyon \\ ${ }^{\circ}$ University of Adana, Turquie
}

\section{Introduction}

Les recherches translinguistiques sur le développement du lexique chez l'enfant dégagent des similitudes dans la trajectoire et la composition du vocabulaire durant la deuxième année de vie de l'enfant. Un des faits observés chez la plupart des enfants est une plus grande utilisation des noms (biais nominal) par rapport aux autres catégories lexicales. Ce phénomène de biais nominal a reçu des explications de nature conceptuelle, linguistique ou contextuelle. Mais, rares sont les travaux à avoir envisagé les explications de manière simultanée sur les mêmes sujets. Par ailleurs, ces études se sont concentrées presqu'exclusivement sur des langues indo-européennes ou asiatiques limitant par la même la généralisation des résultats. Nos travaux tendent à vérifier les explications linguistiques et contextuelles en examinant les caractéristiques du discours adressé à l'enfant dans deux situations de communication (lecture d'album et jeu avec maison de poupée) et deux langues (français et turc).

Dans ce travail, nous cherchons à expliquer la répartition lexicale chez les enfants français et turcs au début du développement. Nous nous intéressons en particulier à la distribution et à la position des verbes et des noms dans l'input maternel. Ces caractéristiques changent-elles ou restent-elles les mêmes en fonction de la situation? Ces caractéristiques sont-elles les mêmes ou sont-elles différentes d'une langue à l'autre?

\section{Le biais nominal et ses explications}

La composition du lexique du très jeune enfant est marquée pour de nombreuses langues par un biais nominal : on rapporte en effet des inventaires contenant plus de noms que de verbes. La domination des noms sur les autres catégories grammaticales pendant la période des premiers mots est observée dans l'étude translinguistique princeps de Gentner (1982) chez des enfants apprenant l'anglais, l'allemand, le japonais, le turc, le kaluli et le chinois mandarin. Des résultats semblables sont répliqués en anglais (voir entre autres, Bornstein et al., 2004 ; Fenson et al., 1994 ; Goldfield, 2000) mais également en italien (Bornstein et al., 2004 ; Caselli et al., 1995 ; Tardif et al., 1997), en hébreu (Bornstein et al., 2004; Dromi, 1987 ; Maital et al., 2000), en espagnol (Jackson-maldonado et al., 1993) et en flamand (Bornstein et al., 2004 ; De Houwer \& Gillis, 1998 ; Gillis \& Verlinden, 1988).

Gentner (1982) propose une explication conceptuelle à cette trajectoire particulière. Elle avance « the Natural Partitions Hypothesis », hypothèse selon laquelle les noms seraient 
acquis avant les prédicats (verbes et adjectifs) car leurs référents sont plus accessibles et plus stables que ceux des prédicats. Cette hypothèse a été progressivement abandonnée avec la mise en évidence de trajectoires acquisitionelles différentes chez des apprenants de chinois mandarin (Tardif, 1996), de coréen (Choi \& Gopnik, 1995 ; Gopnik et al., 1996 ; Cheng, 1994) et de tzotzil (de Leon, 1998). Dans ces études, le biais dans la composition du vocabulaire porte sur le verbe et non pas sur le nom. Tardif (1996) par exemple, rapporte que 9 enfants chinois sur 10, âgés de 22 mois produisent significativement plus de types de verbes différents que de types de noms communs.

L'explication de nature conceptuelle se voit par conséquent progressivement remplacée par une explication linguistique : le biais nominal ou verbal est à mettre en relation avec le type d'input que l'enfant reçoit à travers le langage adressé à l'enfant (LAE). Les différences de fréquence des noms par rapport aux verbes dans le premier lexique des enfants sont expliquées par des caractéristiques particulières de l'input: certaines favorisant la production des noms, d'autres celle des verbes. Les caractéristiques de l'input évoquées dans la littérature (Bornstein et al., 2004 ; Ogura et al., 2006 ; Tardif et al., 1997) sont la fréquence des noms vs verbes, la transparence du système morphologique nominal $v s$ verbal, la position saillante des noms $v s$ verbes ou encore la focalisation particulière des mères sur une catégorie grammaticale plutôt qu'une autre.

Prenons le cas de l'acquisition de l'anglais pour lequel un important biais nominal est rapporté. La position finale (et dans une moindre mesure la position initiale) sont des positions auxquelles les enfants sont particulièrement attentifs (Slobin, 1985). L'ordre des constituants des phrases varie en fonction des langues: dans certaines ce sont les noms qui sont placés en position finale et donc en position saillante, dans d'autres, ce sont les verbes. En anglais, compte tenu d'un ordre des mots SVO plutôt stable, une emphase est placée sur les noms. Par ailleurs dans le langage que les parents anglophones adressent à leur enfant, les noms apparaissent plus fréquemment que les verbes dans les énoncés à un seul mot et tendent à être davantage placés en fin des énoncés longs (Goldfield, 1993 ; Tardif et al., 1997). À l'inverse, certaines propriétés structurales des langues asiatiques favorisent l'emploi des verbes. En chinois mandarin par exemple, les verbes apparaissent plus souvent en fin d'énoncés que les noms. Ce sont donc les caractéristiques syntaxiques des langues comme l'ordre des mots qui rendent certains éléments plus ou moins saillants et qui peuvent expliquer le déséquilibre noms/verbes observé dans les premiers vocabulaires des enfants.

Une explication impliquant l'influence du contexte communicatif sur la composition du vocabulaire vient compléter ce tableau. En effet, seul ce type d'explication permet de rendre compte de résultats divergents obtenus pour une même langue. Le contexte communicatif est entendu ici comme le type d'activité dans laquelle les participants à une conversation sont engagés. Un nombre non négligeable d'études révèlent des différences dans le discours maternel en fonction du contexte de communication (Goldfield, 1993; Leaper \& Gleason, 1996; Lucariello \& Nelson, 1986; O’Brien \& Nagle, 1987). Ceci a conduit les chercheurs à tenir compte de la situation de communication pour l'analyse de l'utilisation des noms et des verbes. En 1993, Goldfield étudie 12 dyades mères-enfants dans deux contextes différents: jeux avec 
jouets et autres jeux. Elle montre que le contexte du jeu avec des jouets focalise l'attention de l'enfant sur les noms dans la mesure où la mère étiquette les objets présents pendant la séance de jeux. Dans le contexte de jeux autres, la mère utilise un nombre plus importants de verbes différents. La production de plus de noms différents dans le contexte de lecture d'album par rapport au contexte de jeux avec des jouets est également un phénomène souvent décrit. Gelman \& Tardif (1998), en comparant le langage adressé à l'enfant au cours d'une lecture partagée avec celui produit lors d'une séance de jeux découvrent que les noms sont les plus nombreux dans la première situation alors que les verbes sont plus nombreux dans la seconde et cela dans les deux langues observées (Anglais et Mandarin). Choi (2000) arrive à la même conclusion chez des mères coréennes.

\section{Comparaison français-turc}

Nous avons décidé de comparer le langage adressé à l'enfant par des mères turques à celui de mères francophones. À notre connaissance, il existe peu de travaux à ce jour qui se soient intéressés à la problématique du développement des noms et des verbes en turc et seulement l'une de ces études (Kern \& Türkay, 2006) a comparé ce développement avec celui des enfants francophones.

Cette absence de travaux est d'autant plus surprenante que les deux langues présentent des différences typologiques extrêmement intéressantes pour le traitement de cette problématique. En effet, le français fait partie des langues que l'on qualifie de «orientées» vers les noms (noun-friendly) alors que le turc présente des caractéristiques typologiques qui le rapprocheraient davantage des langues " orientées » vers les verbes (verb-friendly). L'ordre des mots le plus fréquent en français est l'ordre SVO même s'il existe une certaine flexibilité en français oral (Harris, 1990). Cet ordre des mots favorise les noms puisqu'il les place dans une position particulièrement saillante, à savoir la position finale d'énoncés. La syntaxe du turc tend quant à elle à favoriser la catégorie des verbes. L'ordre des mots canonique en turc est SOV même si des variations sont possibles dans des contextes bien spécifiques (Göksel \& Kerslake, 2005 ; Slobin, 1982). De plus, les syntagmes nominaux peuvent être omis à condition que leurs référents soient clairement identifiables dans le discours (pro-drop). Par ailleurs, les récentes découvertes concernant l'acquisition lexicale en turc et en français confortent également le bienfondé d'une comparaison entre ces deux langues. En effet, il existe tout un ensemble de travaux qui tendent à montrer qu'on retrouve véritablement un biais nominal dans les productions des enfants francophones alors qu'en turc il n'y a pas véritablement de domination d'une catégorie sur l'autre.

Pour ce qui est de l'acquisition lexicale en français les noms sont acquis avant les verbes. Ce constat est basée sur des données naturelles spontanées (Bassano, 2000 ; Bassano et al., 1998 ; Parisse et Lenormand, 2000) et des compte-rendus parentaux (Kern, 2007 ; Kern \& Gayraud, 2010). Parisse et Lenormand (2000) ont enregistré les jeux symboliques de 27 enfants francophones de 2 ans. Ils rapportent un nombre plus important de types de noms que de types de verbes : 29\% de noms différents sont 
produits dans les énoncés à un seul mot et $44 \%$ dans les énoncés de plus d'un mot contre $18 \%$ et $22 \%$ seulement de verbes. Dans une étude longitudinale portant sur une enfant francophone entre 14 et 30 mois ainsi que dans une étude transversale de 12 enfants observés à 12 et à 30 mois, Bassano et al. (1998) montrent que les noms et les items paralexicaux (interjections, particules) sont les types de mots les plus fréquents jusqu'à 20 mois. À 20 mois, émergent des prédicats (verbes et adjectifs) et des mots grammaticaux (articles, prépositions, ...). Par le biais de l'inventaire français du développement communicatif (IFDC), Kern \& Gayraud (2010) confirment ce résultat: entre 8 et 16 mois, $80 \%$ des mots produits sont des noms; entre 16 et 30 mois, les noms sont moins représentés $(58,5 \%)$ compte-tenu de l'augmentation des verbes et des adjectifs.

Les études sur l'acquisition des noms et des verbes en turc se sont penchées plus particulièrement sur la distribution, la fréquence et les propriétés morphologiques et positionnelles de ces éléments. Parmi elles, l'étude de Ketrez \& Aksu-Koç (2003) qui examine le paradigme flexionnel des noms et des verbes chez un enfant turcophone et qui découvre que les deux paradigmes sont acquis au même moment. De la même manière, l'étude longitudinale de Türkay (2005) qui observe 5 enfants pendant 5 ans indique que chez ces sujets les noms sont acquis au même moment que les verbes. Enfin Kern \& Türkay (2006) arrivent à des conclusions quelque peu différentes dans une étude longitudinale et translinguistique de 9 enfants français et turcs. Elles montrent d'une part que les noms (en types et occurrences) sont majoritaires dans la production des enfants français et turcs avant et après explosion lexicale mais que la différence entre les deux catégories est moins grande en turc qu'en français.

\section{Données}

Nous disposons d'enregistrements vidéo de 20 enfants francophones monolingues âgés de 16 à 34 mois (âge moyen : 23 mois) et de 20 enfants turcophones monolingues âgés de 16 à 32 mois (âge moyen : 24 mois) en interaction avec leurs mères. Toutes les mères ont un niveau d'études universitaires et travaillent à plein temps. Les familles vivent à Lyon pour les francophones ou à Adana pour les turcophones dans un contexte linguistique monolingue. Les enfants francophones (11 filles et 9 garçons) et turcophones (10 filles et 10 garçons) ont un développement physique, intellectuel et social conforme à la norme nationale.

Les sujets ont été filmés au domicile des parents en interaction avec leur mère lors de deux situations différentes : la lecture partagée d'un album d'images sans texte et un jeu avec une maison de poupées. Chaque situation dure 10 minutes. L'album (The Farm Picture Book, Amery \& Cartwright, 1986) utilisé pour éliciter la lecture partagée contient 5 scènes de ferme qui représentent de nombreux personnages impliqués dans des événements variés. Au cours de la session de jeu, les dyades mère-enfant utilisent une maison miniature composée de plusieurs pièces et d'objets mobiles (personnages, frigidaire, télévision, miroir, etc.). Dans les deux situations, les mères ont pour consigne de se comporter le plus naturellement possible. 
Tous les énoncés des mères et des enfants ont été transcrits orthographiquement et saisis sous le logiciel CLAN (MacWhinney, 2000) par des locuteurs natifs du français ou du turc. Un codage a été réalisé sur la production des mères.

\section{Codage}

\subsection{Mesures lexicales}

Les noms ont été codés en deux catégories : noms propres et noms communs. Les vocatifs ont été exclus de l'étude. En ce qui concerne les verbes, seuls les verbes principaux ont été pris en compte : pas d'auxiliaires, de copules, ou de verbes pour attirer l'attention de l'enfant. Ensuite, nous avons calculé le ratio de types de noms par rapport au nombre total de types de noms et de verbes où N-type renvoie au nombre de noms différents et V-type au nombre de verbes différents) et le ratio pour les occurrences où N-occ renvoie au nombre total de noms et V-occ au nombre total de verbes).

\subsection{Mesures syntaxiques}

Les éléments constituant les énoncés ont été codés selon leur catégorie grammaticale, de sorte que pour chaque énoncé on puisse ensuite obtenir la longueur de l'énoncé et la position des noms et des verbes dans l'énoncé.

La longueur des énoncés a été extraite en excluant les interjections et communicateurs (exclamations telles que $O h$ !, $A h$ !, phatiques, ex. hein, marques pour attirer l'attention, ex. regarde! tiens, tu vois). Nous avons ensuite comptabilisé les énoncés à un mot et les énoncés multimots pour vérifier si un contexte ou une langue attire davantage d'énoncés à un mot.

La position des noms et des verbes dans les énoncés a ensuite été extraite, toujours en excluant les interjections et communicateurs.

\section{Résultats}

Nous présentons dans un premier temps les caractéristiques principales de nos données. Nous présentons dans un deuxième temps les mesures lexicales sur les deux langues en fonction des contextes communicatifs (Lecture d'Album - LA et jeu avec la Maison de Poupée - MP) : Ratio_typ et Ratio_occ. Nous nous intéressons ensuite aux mesures syntaxiques, à savoir la longueur des énoncés et la position des noms et des verbes en fonction des langues et des contextes communicatifs (CC).

Les analyses statistiques employées pour obtenir les résultats présentés en Figures 1, 2 et 5 sont des analyses de variance à mesures répétées avec la langue comme variable inter-sujets et le contexte comme variable intra-sujets. Pour les résultats présentés dans les Figures 3, 4, 6, 7 le test $t$ de Student a été utilisé. Pour les graphiques 8 et 9, les analyses statistiques employées sont des analyses de variance à mesures répétées avec la 
langue comme variable inter-sujets et le contexte comme variable intra-sujet.

Nous disposons de 80 enregistrements de 10 minutes chacun, dont 40 en français et 40 en turc (20 enregistrements par CC). Comme l'illustre la Figure 1, le nombre moyen d'énoncés est comparable entre les langues et les CC. Dans LA, les mères françaises produisent 175,5 énoncés ( $\mathrm{DS}=41,5$, min 113 - max 239) et les mères turques 178,1 $(\mathrm{DS}=36$, min 128 - 248). Dans MP, les mères françaises et les mères turques produisent respectivement $175,8(\mathrm{DS}=50,8 ; \min 87-\max 288)$ et 182,3 énoncés $(\mathrm{DS}=50,8 ; \min$ $82-\max 244)$.

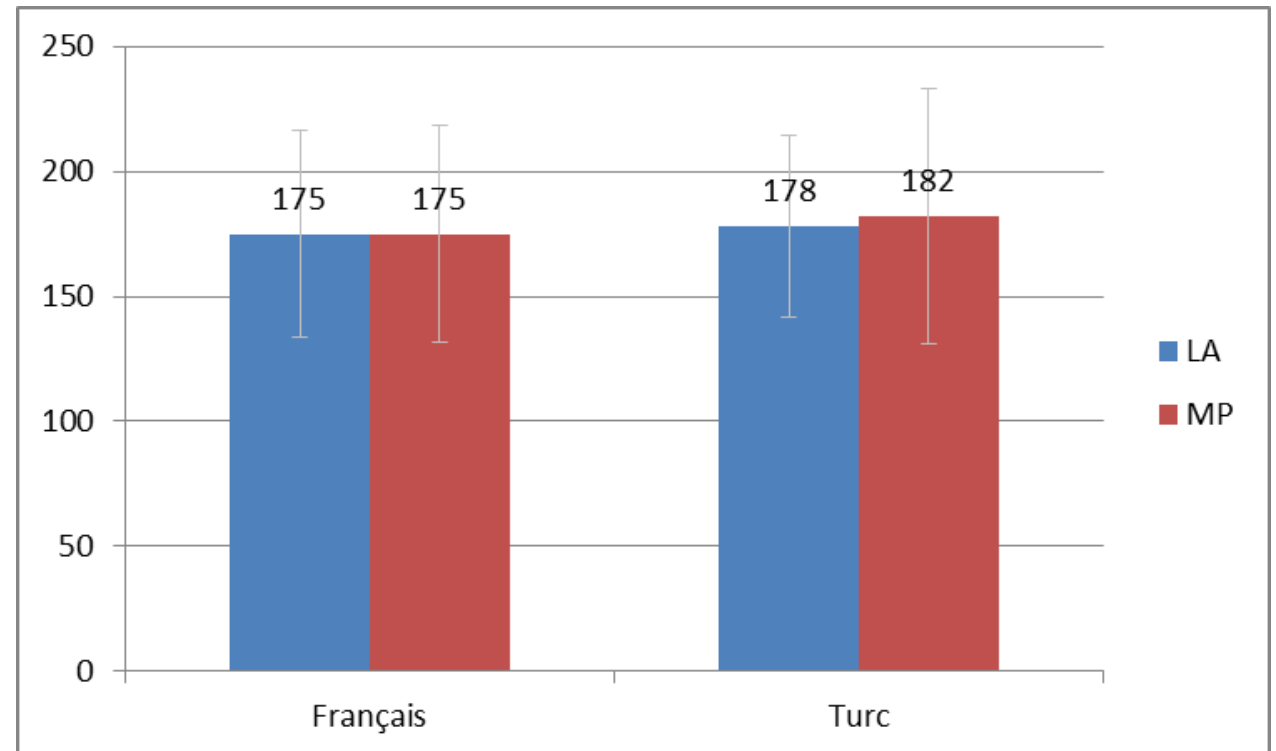

Figure 1. Nombre moyen d'énoncés en fonction de la langue et des contextes communicatifs

La Figure 2 présente le ratio de types de noms en fonction de la langue et des CC. Seul le facteur contexte est significatif. Pour les deux langues, le contexte LA est plus propice aux noms (types) qu'aux verbes et le contexte MP plus propice aux verbes qu'aux noms (effet du contexte, p. $<0,001$ ). Les deux langues confondues on obtient un ratio de 0,454 dans MP (moyenne de 0,456 et 0,452) contre 0,523 (moyenne de 0,527 et $0,519)$ dans LA. 


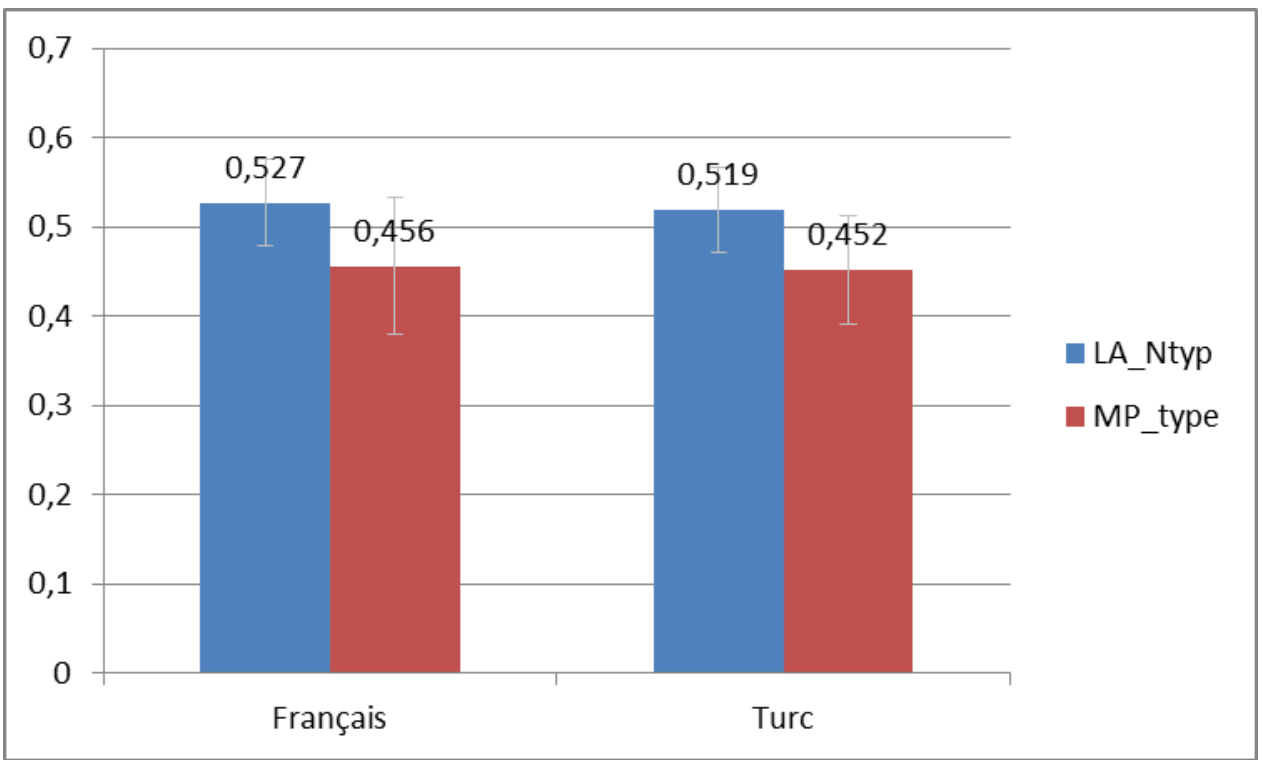

Figure 2. Ratio types de noms en fonction de la langue et des contextes communicatifs

La Figure 3 présente les types de noms et de verbes en fonction de la langue en contexte MP. La prédominance des types de verbes dans le discours adressé à l'enfant dans ce $\mathrm{CC}$ est statistiquement significative pour les deux langues ( $\mathrm{p} .<0,0033$ pour le français et p. $<0,0067$ pour le turc). En contexte MP les mères françaises produisent 45,15 noms différents contre 38,15 verbes alors que les mères turques en produisent 29,9 contre 25 .

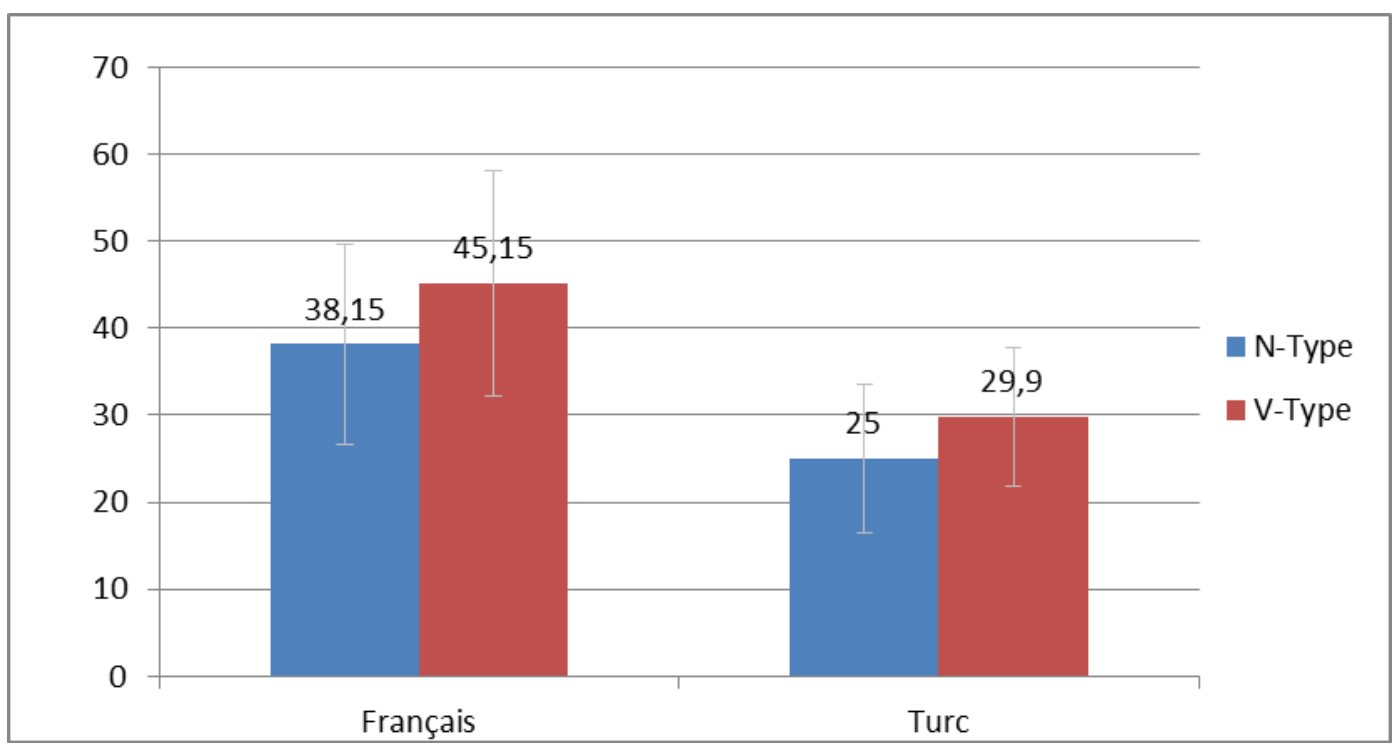

Figure 3. Types de noms et verbes en fonction de la langue en contexte MP

La Figure 4 présente les types de noms et de verbes en fonction de la langue en contexte LA. La prédominance des types de noms dans le discours adressé à l'enfant dans ce CC est statistiquement significative seulement pour le français $(\mathrm{p} .<0,0083)$. Les mères 
françaises utilisent 47 noms différents contre 41 verbes différents. En turc, il y également plus de noms différents que de verbes mais le test $\mathrm{t}$ de Student ne révèle qu'une tendance $(\mathrm{p} .<0,0794): 30,75$ noms contre 28,5 verbes

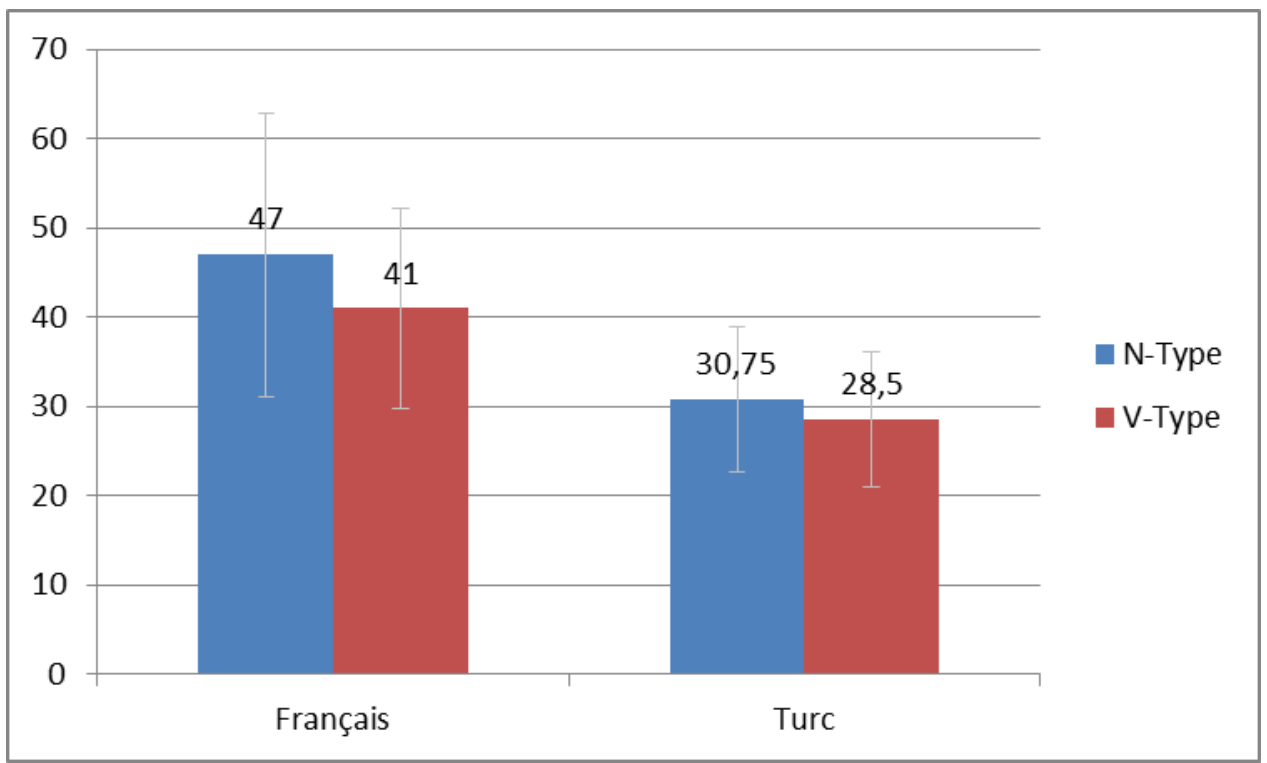

Figure 4. Types de noms et verbes en fonction de la langue en contexte LA

La Figure 5 présente le ratio des occurrences de noms en fonction de la langue et des CC. Pour les deux langues, le contexte LA est plus propice aux noms (occurrences) qu'aux verbes. En français on observe un N_occ de 0,547, en turc de 0,535. Le contexte MP quant à lui est plus propice aux verbes qu'aux noms (effet du contexte, p. $<0,0001$ ) avec un N_occ de 0,455 en français et de 0,432 en turc. Les deux langues combinées, le ratio N_occ est de 0,541 dans LA contre seulement 0,443 dans MP.

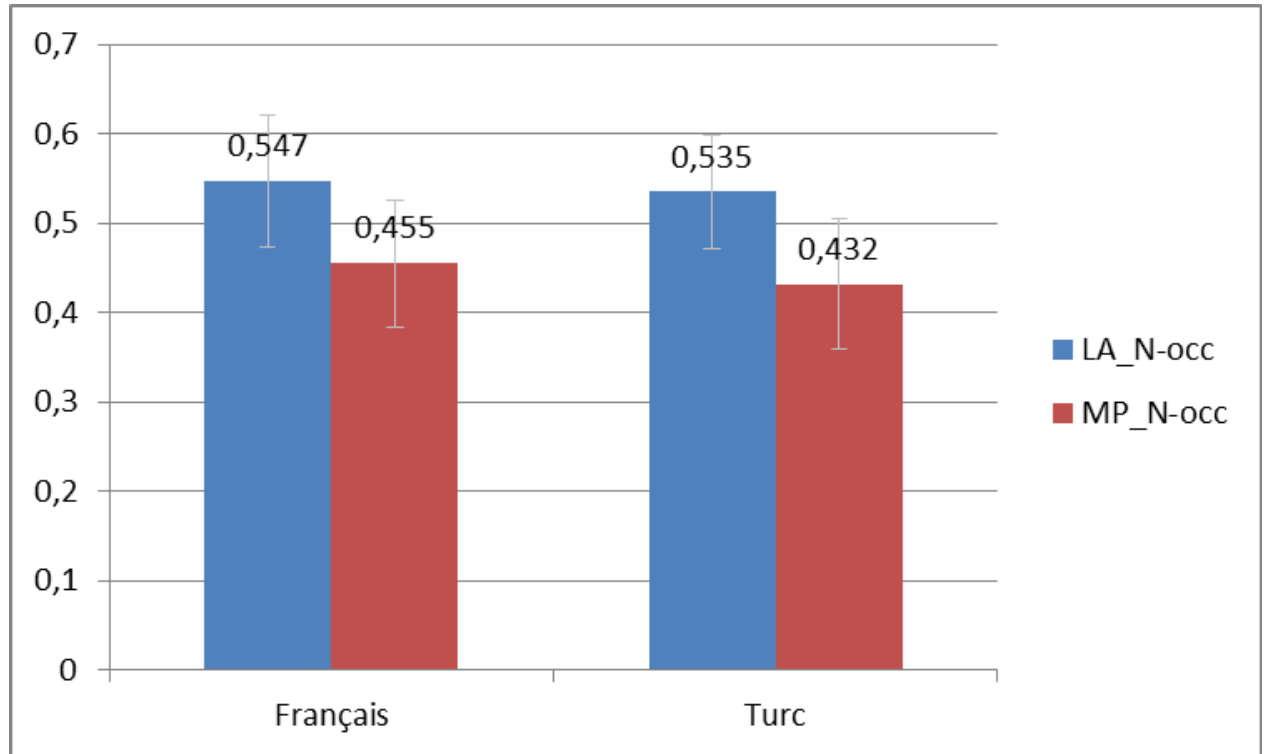


Figure 5. Ratio des occurrences de noms en fonction de la langue et des contextes communicatifs

La Figure 6 présente les occurrences de noms et verbes en fonction de la langue en contexte MP. La prédominance des occurrences de verbes dans le discours adressé à l'enfant dans ce CC est marquée pour les deux langues (p. $<0,0011$ pour le français et p. $<0,0036$ pour le turc). 135,5 verbes contre 102,5 noms sont produits en français, 129 , 75 verbes contre 100,4 noms en turc.

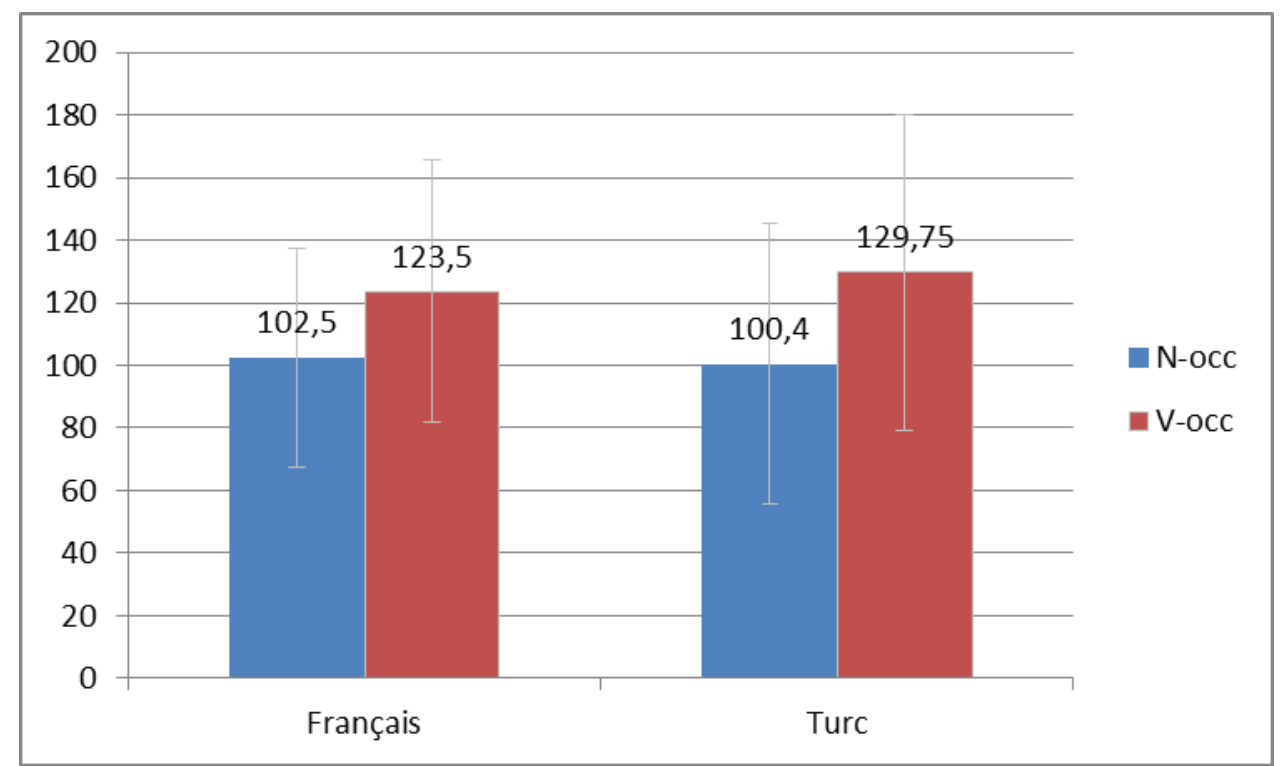

Figure 6. Occurrences de noms et verbes en fonction de la langue en contexte MP

La Figure 7 présente les occurrences de noms et verbes en fonction de la langue en contexte LA. La prédominance des occurrences de noms dans le discours adressé à l'enfant dans ce CC est marquée pour les deux langues (p. $<0,004$ pour le français et p. $<0,0502$ pour le turc). On remarque néanmoins qu'en turc la différence n'est que marginalement significative. En français, les mères produisent 124,9 noms contre 103,25 verbes seulement; en turc on trouve 108,42 noms contre 96,68 verbes. 


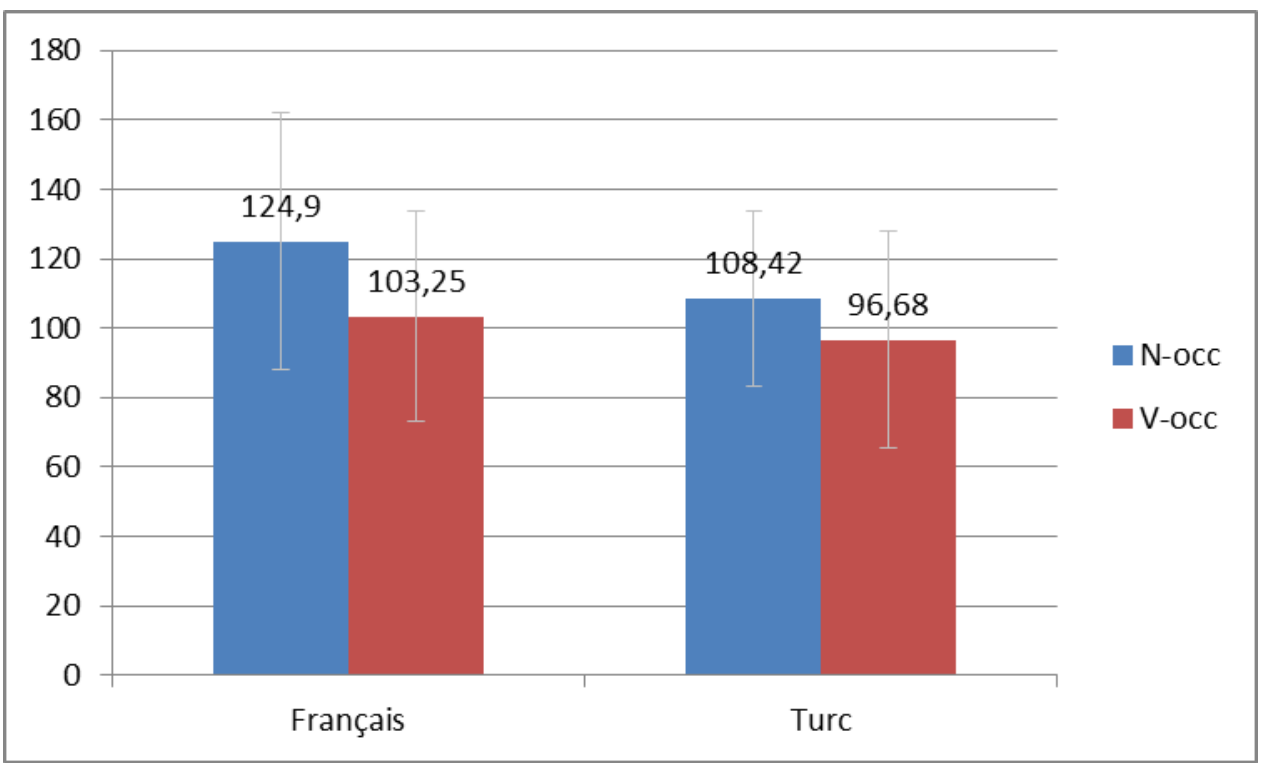

Figure 7. Occurrences de noms et verbes en fonction de la langue en contexte LA

La Figure 8 présente la longueur des énoncés en fonction du contexte et de la langue. On obtient un effet significatif pour les deux facteurs (p. <0,0001). En français et en turc et pour les deux contextes la proportion d'énoncés à plusieurs mots est bien plus élevée que celle des énoncés à un mot, l'écart étant moins important en turc qu'en français. Pour le turc, la proportion d'énoncés à un mot est plus importante en contexte LA que MP (29,8\% vs. 19,34\%). 


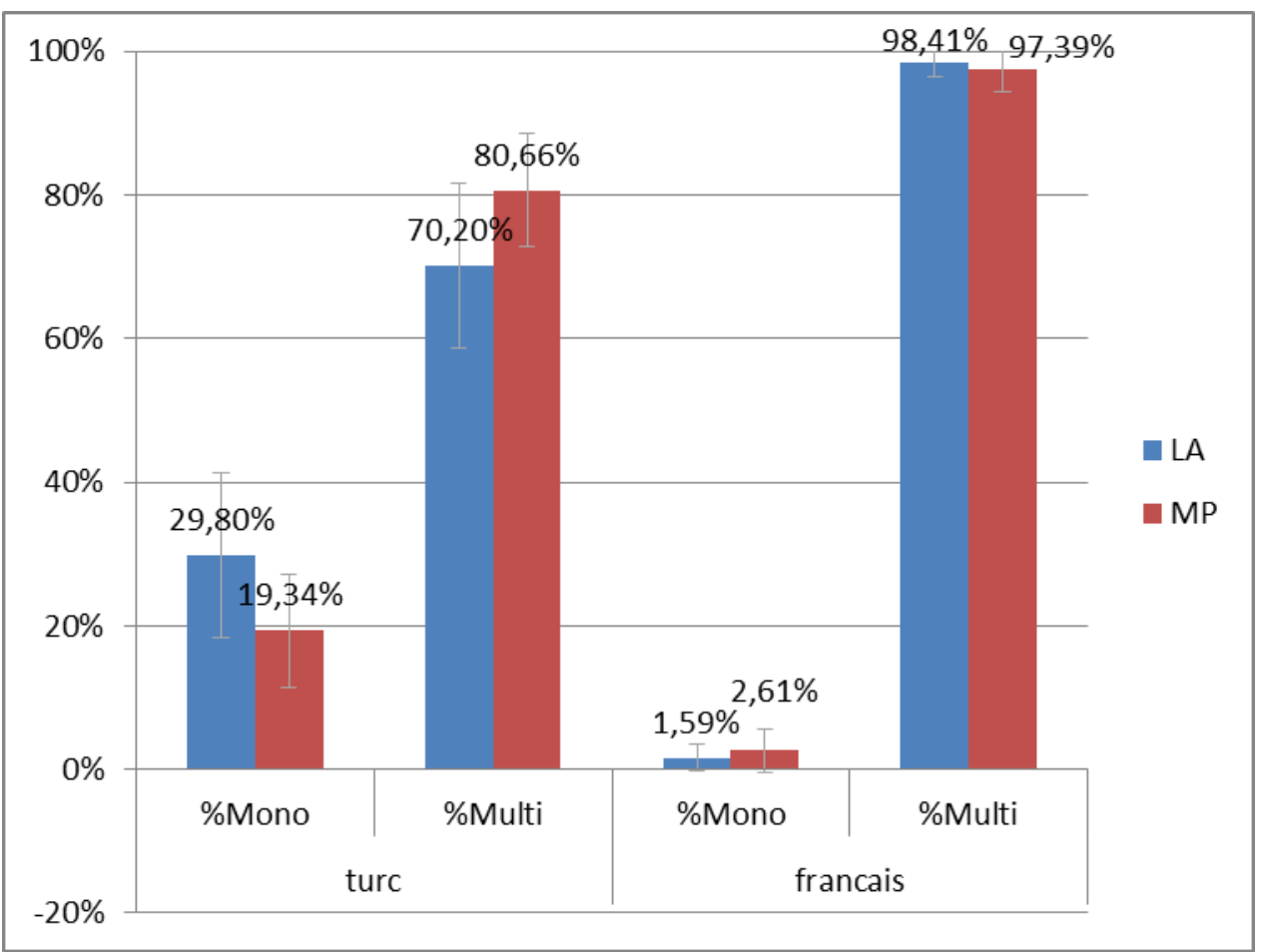

Figure 8. Pourcentage moyen d'énoncés à un mot (Mono) et d'énoncés à plusieurs mots (Multi) en fonction du contexte communicatif et de la langue

La Figure 9 présente la catégorie en position finale en fonction du contexte et de la langue. Les facteurs langue et contexte ont un effet significatif (p. $<0,001)$. En français, la position finale est davantage occupée par des noms que par des verbes. On note davantage de noms en finale en français dans le contexte LA $(59,87 \%)$ que dans le contexte MP (47,61\%). En turc, la position finale est davantage occupée par des verbes que par des noms dans les deux contextes, une légère préférence pour les verbes en finale apparaissant dans le contexte MP (36,46\% en MP contre $28,26 \%$ en LA). 


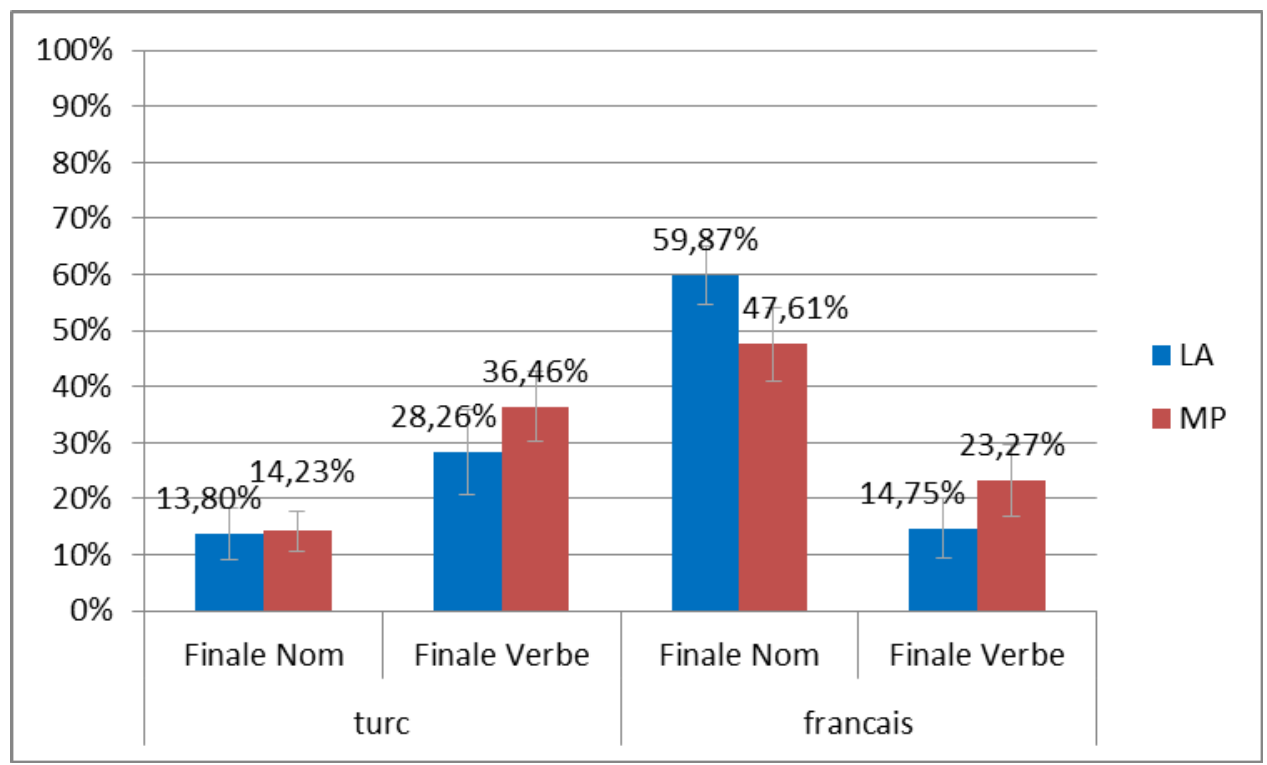

Figure 9. Pourcentage moyen d'éléments de catégorie nom ou verbe en position finale en fonction du contexte et de la langue

\section{Discussion}

Seule la variable contextuelle joue un rôle sur les mesures lexicales, à savoir sur la distribution des noms et des verbes. Dans les deux langues, on observe plus de noms que de verbes dans la lecture d'album (LA) que dans le jeu avec la maison de poupée (MP), et ce, que le type ou les occurrences de noms soient considérés. Dans la maison de poupée on trouve un effet inverse avec une domination des verbes. Ce résultat confirme l'importance du contexte sur la répartition des noms et des verbes dans le langage adressé à l'enfant. Il est très proche des résultats obtenus pour le langage adressé à l'enfant en mandarin, japonais, anglais ou encore coréen. Dans une étude translinguistique, Tardiff et al. (1999) ont comparé le nombre de noms différents à celui des verbes différents dans le langage adressé à l'enfant en mandarin et en japonais. Ils observent dans les deux groupes une préférence pour les noms dans la lecture d'album et la tendance inverse dans les jeux avec des jouets. Ce comportement est le même que celui présent chez nos mères turques et françaises. Cependant la différence entre les noms et les verbes est beaucoup plus marquée : le ratio n-type est de 0,30 dans les jeux avec jouets et de 0,55 dans la lecture d'album en mandarin ; 0,33 et 0,55 en japonais ; 0,46 et 0,53 en français et 0,45 et 0,52 en turc. Un effet significatif du contexte pour les mères japonaises (Ogura et al., 2006) et les mères coréennes (Choi, 2000) a également été trouvé avec un nombre plus important de types et d'occurrences de noms que de verbes dans un contexte de lecture d'album.

Un très léger effet de la langue sur les mesures lexicales apparaît lorsque les deux contextes sont considérés de manière séparée. Ainsi l'étude du contexte de lecture d'album seul fait émerger une nette préférence des noms (types et occurrences) au détriment des verbes chez les mères françaises alors que la différence n'est que 
marginalement significative chez les mères turques. Ainsi dans un contexte tel que la lecture d'album, contexte qui généralement attire les noms la distribution entre noms et verbes est plus équilibrée en turc qu'en français.

Pour ce qui est des mesures syntaxiques, la langue et le contexte influencent significativement les résultats. Quelle que soit la langue, les énoncés multimots dominent sur les énoncés monomots. On peut néanmoins observer un écart moins important entre les deux catégories en turc qu'en français. Cette tendance peut s'expliquer par le fait que le turc étant une langue agglutinante, la proportion d'énoncés composés d'un seul mot est plus importante qu'en français. La répartition des noms et des verbes en position finale est elle aussi contrainte par les facteurs langue et contexte. En effet, en français, ce sont les noms que l'on trouve majoritairement en position finale et ce, en particulier dans le contexte de lecture d'album alors qu'en turc on trouve majoritairement des verbes en position finale et tout particulièrement dans le contexte de la maison de poupée. Ce dernier résultat rejoint celui de Slobin (1982) qui montre qu'en turc $69 \%$ des énoncés parentaux finissent par un verbe.

\section{Conclusion}

Nous avons essayé de comprendre les spécificités du développement lexical en français et en turc, à savoir la présence d'un biais nominal important en français et celui d'un biais nominal plus discuté en turc. Pour ce faire nous avons testé les hypothèses linguistiques et contextuelles en observant le langage adressé à l'enfant français et turc dans deux contextes de communication.

Ce travail montre une influence plus importante du facteur contexte que du facteur langue sur les mesures lexicales. Les noms sont plus diversifiés et plus fréquemment produits que les verbes dans le contexte de lecture d'album dans les deux langues même si la différence nom/verbe est moins importante en turc. Ce phénomène est un phénomène récurrent même dans les langues considérées comme orientées vers les verbes. Par contre, on note une interaction entre les facteurs contexte et langue sur les mesures syntaxiques et en particulier sur les caractéristiques positionnelles des noms et des verbes : plus de noms en position finale dans la lecture d'album contre plus de verbes en turc dans la situation de maison de poupée.

Ce travail montre également que les noms sont mis en avant dans le discours adressé à l'enfant en français. Les noms dominent et ce, quelle que soit la situation de communication. Ce résultat concorde avec ceux de Parisse \& LeNormand (2000) et Poulin-Dubois et al. (1995) qui ont observé dans le langage adressé à l'enfant français (en France et au Canada) une domination des noms sur les verbes. La situation est moins nette dans le discours adressé à l'enfant en turc : sur un continuum allant du plus orienté vers les noms au moins orienté vers les noms, le turc pourrait être situé entre des langues très orientées vers les noms comme le français ou l'anglais et des langues asiatiques telles que le coréen ou le chinois mandarin que l'on considère comme très orientées vers les verbes. 
Un des prolongements à ce travail sera l'étude des caractéristiques morphosyntaxiques de l'input maternel. En effet, Il existe de grandes différences dans la diversité, la transparence et la régularité du marquage morphologique entre les deux langues. Le système morphologique des verbes et des noms est plus pauvre en français qu'en turc. En français, les noms portent seulement les marques de nombre (singulier et pluriel) et du genre (féminin et masculin), alors qu'en turc, les noms sont marqués pour le nombre, le cas (accusatif, datif, locatif, ablatif et génitif) et la possession $\left(1^{\text {ere }}, 2^{\text {ème }}\right.$ et $3^{\text {ème }}$ personne, singulier et pluriel). Les verbes en turc sont encore davantage marqués que les noms. On y trouve des marques de personne, nombre, temps, aspect, modalité, voix, négation et interrogation. Les verbes en français ne sont marqués que pour le nombre, le temps et le genre. Par contre, contrairement au français, le système morphologique du turc est transparent et régulier.

Le biais nominal présent dans le développement de l'enfant français et le biais nominal atténué dans celui de l'enfant turc sont explicables par les caractéristiques fréquentielles et positionnelles des noms et des verbes dans le langage adressé à l'enfant. Mais ces caractéristiques ne sont pas suffisantes. En effet pour expliquer la trajectoire acquisitionnelle des enfants, il faut considérer le facteur contexte de communication comme un facteur à part entière et l'introduire comme facteur à part entière dans le modèle explicatif. Ainsi la répartition nom/verbe dans les productions enfantines devrait égalment être expliquée à l'aune des caractéristiques situationnelles de production.

\section{Références bibliographiques}

Amery, H. \& Cartwright, S. (1986). The Farm Picture Book. Macmillan.

Bassano, D. (1998). Sémantique et syntaxe dans l'acquisition des classes de mots : l'exemple des noms et des verbes en français. Langue Française, 118, 26-48.

Bassano, D. (2000). Early development of nouns and verbs in French: exploring the interface between lexicon and grammar. Journal of Child Language, 27, 521-559.

Bassano, D., Maillochon, I. \& Eme, E. (1998). Developmental changes and variability in early lexicon: A study of French children's naturalistic productions. Journal of Child Language, 25, 493-531.

Bornstein, M. H., Cote, L. R., Maital, S., Painter, K., Park, S., Pascual, L., Pêcheux, M., Ruel, J., Venuti, P. \& Vyt, A. (2004). Cross-Linguistic Analysis of Vocabulary in Young Children: Spanish, Dutch, French, Hebrew, Italien, Korean, and American English. Child Development, 4, 1115-1139.

Caselli, M. C., Bates, E., Casadio, P., Fenson, J., Fenson, L., Sanderl, L. \& Weir, J. (1995). A cross linguistic study of early lexical development. Cognitive Development, 10(2), 159-200.

Cheng, S. W. (1994). Beginning words of three children acquiring Mandarin Chinese. Manuscript, National Chung Cheng University. Taiwan.

Choi, S. (2000). Caregiver input in English and Korean: use of nouns and verbs in book reading and toy play contexts. Journal of Child Language, 27, 69-96.

Choi, S. \& Gopnik, A. (1995). Early acquisition of verbs in Korean: a cross-linguistic study. Journal of Child Language, 22, 497-527.

De Houwer, A. \& Gillis, S. (1998). Dutch child language: an overview. In Gillis, S. \& De Houwer, A. (éd.), The acquisition of Dutch, Amsterdam: John Benjamins, 1-100.

de León, L. (1998). The emergent participant: Patterns of socialization of Tzotzil (Mayan) infants. Journal of Linguistic Anthropology, 8(2), 131-161. 
Dromi, E. (1987). Early Lexical Development. Cambridge: C. U. P.

Fenson, L., Dale, P. S., Reznick, J. S., Thal, D., Bates, E., Hartung, J. P., Petnick, S.\& Reilly, J. S. (1993). MacArthur Communicative Development Inventories. San Diego, CA: Singular Publishing Group.

Fernald, A., \& Morikawa, H. (1993). Common themes and cultural variations in Japanese and American mothers' speech to infants. Phonetica, 57, 242-254.

Gelman, S. \& Tardif, T. (1998). Acquisition of nouns and verbs in Mandarin and English: The Proceedings of the Twenty-ninth Annual Child Language Research Forum, Stanford, 27-36.

Gentner, D. (1982). Why nouns are learned before verbs: linguistic relativity versus natural partitioning. In Kuczaj, S. A. (éd.), Language Development, Vol. 2: Language, Thought and Culture, Hillsdale, NJ: Erlbaum, 301-334.

Gillis, S., \& Verlinden, A. (1988). Nouns and early verbs in early lexical development: Effects of input frequency? Antwerp Papers of Linguistics, 54. Antwerp: Antwerp University Press.

Göksel, A. \& Kerslake, C. (2005). Turkish: a comprehensive grammar. Routledge.

Goldfield, B. (1993). Noun bias in maternal speech to one-year olds. Journal of Child Language, 20, 85-99.

Goldfield, B. A. (2000). Nouns before verbs in comprehension vs. production: the view from pragmatics. Journal of Child Language 27, 501-20.

Gopnik, A., Choi, S. \& Baumberger, T. (1996). Cross-linguistic differences in semantic and cognitive development. Cognitive Development, 11(2), 197-227.

Harris, M. (1990). French. In: Comrie, B. (éd.), The world's major languages, Oxford: Oxford Univ. Press, 210-235.

Jackson-Maldonado, T. D., Marchman, V., Bates, E. \& Gutienez-Clennen, V. (1993). Early lexical development in spanish-speaking infants and toddlers. Journal of Child Language, 20(3), 523-549.

Kern, S., (2007). Lexicon development in French-speaking infants. First Language, 27 (3), 227-250.

Kern, S. \& Türkay, F. N. (2006). Influence cognitive générale et/ou langagière spécifique sur les premiers mots des enfants: étude pilote chez des enfants français et des enfants turcs. Poster presented at ARCO.

Kern, S. \& Gayraud, F. (2010). Inventaire Français du Développement Communicatif. Grenoble : Éditions La Cigale.

Ketrez, N. \& Aksu-Koç, A. (2003). Acquisition of noun and verb categories in Turkish. In Özsoy, A.S., Akar, D. Nakıpoğlu Demiralp M., Erguvanl1-Taylan, E. \& Aksu-Koç, A (Eds.), Proceedings of the Tenth International Conference in Turkish Linguistics, 239-45, İstanbul: Boğaziçi University Press.

Kim, M., McGregor, K. K. \& Thompson, C. K. (2000). Early Lexical Development in English and Korean speaking children: language-general and language-specific patterns. Journal of Child Language, 27, 225-254.

Leapar, C. \& Gleason, J. B. (1996). The relationship of play activity and gender to parent and child sex-typed communication. International Journal of Behavioral Development 19, 689-703.

Lucariello, J. \& Nelson, K. (1986). Context effects on lexical specificity in maternal and child discourse. Journal of Child Language 13, 507-22.

MacWhinney, B. (2000). The CHILDES Project: Tools for Analyzing Talk. Volume 1: Transcription format and programs. Volume 2: The Database. Mahwah, NJ: Lawrence Erlbaum Associates.

Maital, S. L., Dromi, E., Sagi, A. \& Bornstein, M. H. (2000). The Hebrew Communicative Development Inventory: language specific properties and cross-linguistic generalizations. Journal of Child Language, 27, 43-67.

O'Brien, M. \& Nagle, K. J. (1987). Parents' speech to toddlers: the effect of play context. Journal of Child Language 14 (2), 269-79.

Ogura, T., Dale, P. S., Yamashita, Y., Murase, T. \& Mahieu, A. (2006). The use of nouns and verbs by Japanese children and their caregivers in book-reading and toy-playing contexts. Journal of Child Language, 33, 1-29.

Parisse, C. \& Le Normand, M. T. (2000). How children build their morphosyntax: the case of French. Journal of Child Language, 27(2), 267-292.

Poulin-Dubois, D., Graham, S. \& Sippola, Z. (1995). Early lexical development: the contribution of parental labelling and infants categorization skills abilities. Journal of Child Language, 22, 325-343. 
Slobin, D. I. (1982). Universal and particular in in the acquisition of language. In Wanner, E. \& Gleitman, L. R. (Eds.), Language Acquisition: the state of art, Cambridge: Cambridge University Press.

Slobin, D. (1985). Cross linguistic study of language acquisition. Hillsdale, NJ: Lawrence Erlbaum Associates.

Tardif, T. (1996). Nouns are not always learned before verbs: evidence from Mandarin speakers'early vocabularies. Developmental Psychology, 32, 491-504.

Tardif, T., Gelman, A. S. \& Xu, F. (1999). Putting the Noun Bias in Context: A Comparison of English and Mandarin. Child Development, 70(3), 620-635.

Tardif, T., Shatz, M., \& Naigles, L. (1997). Caregiver speech and children's use of nouns versus verbs: A comparison of English, Italian and Mandarin. Journal of Child Language, 24, 535-565.

Türkay, F. N. (2005). Children's Early Lexicon in terms of Noun/Verb Dominance. Unpublished Doctoral Dissertation. Çukurova University.

Choi, S., \& Gopnik, A. (1995). Early Acquisition of Verbs in Korean: A Crosslinguistic Study. Journal of Child Language, 22, 497-529.

Ogura, T., Dale, P. S., Yamashita, Y., Murase, T. \& Mahieu, A. (2006).The use of nouns and verbs by Japanese children and their caregivers in book-reading and toy-playing context, Journal of Child Language 33, 1-29. 